SHS Web of Conferences 24, 01009 (2016)

DOI: $10.1051 /$ shsconf/20162401009

(C) Owned by the authors, published by EDP Sciences, 2016

\title{
Investigation on nursing service satisfaction of the elderly living in nursing home and influencing factors--Taking Zhengzhou City as an example
}

\author{
Wenhui Xing*, Ruimin Fu, Ding Wang \& Huiping Chang \\ Department of Social Demography, Henan Institute of Education, Zhengzhou, Henan, China
}

\begin{abstract}
Objective: Investigate the status quo of the elderly living in the nursing home and their satisfaction and needs of nursing services, and analyze the service needs of the elderly living in the nursing home of Zhengzhou City. Methods: Randomly select 312 elderly people from 84 nursing homes for questionnaire survey and in-depth interview. Results: More than $60 \%$ of the respondents show their satisfaction on the current life in the nursing home. Sex, occupation, physical condition, dietary level, accommodation level, service level and health care conditions of the nursing home have a significant impact on the satisfaction of the elderly. Conclusion With the growth of the aging of population, improving the level of service for the elderly and improve the service facilities is an essential way to improve the satisfaction and urgent needs of the elderly.
\end{abstract}

Keywords: nursing home; the elderly; satisfaction; influencing factors

\section{INTRODUCTION}

With the constant growth of the aging population, China is experiencing the aging process with the largest scale and the fastest speed in history. The population and urbanization sampling survey data published by Zhengzhou Municipal Bureau of Statistics in 2014 show that the population with an age which is greater than or equal to 65 years old is 816,000 , accounting for $8.71 \%$ of the total population of Zhengzhou City. It is significantly higher than the aging standard of the United Nations (7\%). As the most populous city in the Central China, whether the pension problems of Zhengzhou City can be properly addressed or not directly affects the harmony and stability of the whole province and the whole country. This research adopts the field survey and interview methods to survey the satisfaction of the elderly in the nursing home of Zhengzhou City on the status quo of life, and analyze the factors affecting the satisfaction of the elderly, so as to provide a theoretical basis for improving their quality of life.

*Corresponding author: xingwenhui429@126.com

\section{RESEARCH OBJECTS AND METHODS}

The research group adopts self-designed questionnaire and randomly selects 312 elderly people from 84 nursing homes to do questionnaire survey in Erqi District, Zhongyuan District, Jinshui District, Huiji District and Guancheng Hui District in Zhengzhou City from April 2013 to April 2015. There are 29 questions in the questionnaire, involving in the basic situation of the elderly, the living conditions of the elderly and the satisfaction of the elderly on the nursing home. In addition, an interview outline is designed to do the face-to-face and in-depth interviews with the elderly in a way of chat in order to understand the living conditions of the elderly which include occupancy reasons, suggestions and wishes on the nursing home and the local government, keep records of the attitude and expression of answering questions by the elderly, and deeply explore the inner world of the elderly.

312 copies of questionnaires are issued, and 312 copies are recovered. There are 302 valid questionnaires with the recovery rate of $100 \%$, and the effective recovery rate of $96.79 \%$. WPS table is used to input data, and the statistical software is used for descriptive analysis. Moreover, we conduct $\mathrm{X}^{2}$ test for the factors affecting the satisfaction of the elderly in 
order to meet $\mathrm{P}<0.05$, with difference and statistical significance.

\section{RESULTS}

\subsection{Basic situation of the research objects}

Among the respondents, the male population accounts for $45.4 \%$, and the female population accounts for $54.6 \%$; the population with an age of over 80 accounts for $56.5 \%$; the population with an age of 70 to 79 accounts for $31.5 \%$; the population with an age of 60 to 69 accounts for $7.4 \%$; the population with an age of 50 to 59 accounts for $4.6 \%$; viewing from the degree of education, the illiterate population accounts for $25.9 \%$, population with the degree of primary school accounts for $23.1 \%$, population with the degree of junior high school accounts for $23.1 \%$, population with the degree of senior high school and technical secondary school accounts for $15.7 \%$, population with the degree of junior college and undergraduate accounts for $8.3 \%$, population with the degree of master and doctor accounts for $0.9 \%$; viewing from the physical condition, the population who can completely take care of themselves accounts for $73.1 \%$, who can partly take care of themselves accounts for $25.9 \%$, and who could not take care of themselves accounts for $0.9 \%$; viewing from the occupation before entering into the nursing home, the unemployed account for $15.7 \%$, the farmers account for $17.6 \%$, the self-employed account for $5.6 \%$, the enterprise' retirees account for $25.9 \%$, and the public institution's retirees account for $31.4 \%$.

\subsection{Analysis of the satisfaction of the elderly in the nursing home}

\subsubsection{Impact of the basic situation of the elderly on satisfaction}

For the question that "Are you satisfied with your life in the nursing home", the population who are "satisfied", "basically satisfied", "not satisfied" and "helpless or indifferent" accounts for $61.1 \%, 23.1 \%, 6.5 \%$ and $9.3 \%$ respectively. It indicates that the subjective happiness of the elderly in the nursing home of Zhengzhou City is higher, which is consistent with some domestic research results ${ }^{[1,2]}$. This is because the quality of people today constantly improves, the social morality of respecting the old and cherishing the young continues to strengthen, and all walks of life give more emphasis on the problem of the aging. What's more, the elderly who are open-minded, optimistic and have filial children and fixed economic sources can keep a more positive attitude towards life and wishfully get along with others.

Compared with the elderly with different sexes, the satisfaction rate of the female respondents (which includes "satisfied" and "basically satisfied", and hereinafter the same) is $76.3 \%$, and that of the male respondents is $93.9 \%$. The satisfaction rate of the male population is higher than that of the female population with a significant difference $(\mathrm{P}<0.05)$, which may be because the female emotional life is more rich, delicate, sensitive and vulnerable to be affected by the external environment, such as meals, accommodation, nursing staff of the institution and so on.

Compared with the elderly with different physical conditions, the satisfaction rate of the elderly who can completely take care of themselves accounts for $89.9 \%$, who can partly take care of themselves accounts for $71.1 \%$ with a significant difference ( $\mathrm{P}$ $<0.01$ ). It indicates that the happiness of the elderly is affected by the health condition. The better the health condition of the elderly is, the higher the happiness level is, which is consistent with the related reports ${ }^{[3]}$. The reasons are as follows: 1) In 84 nursing homes surveyed, 48 of them explicitly stipulate that, the elderly who can partly take care of themselves are not allowed to leave the residence, and those who can leave should be accompanied by their family only. However, most of the elderly live in the nursing home, because their children are busy at work and have no time. Therefore, the elderly who can partly take care of themselves are unable to have free activity, inevitably leading to be melancholy.

2) The elderly who can partly take care of themselves require being cared by the nursing staff. The interview finds that, the nursing staff work 10 hours a day. In addition to giving injections, dispensing medicines, health education and some medical care, they also help the elderly turn over, wash, go to the toilet, and take meals or do other nursing work ${ }^{[4]}$. However, the nursing staffs are tired with a small number and low wages, so they are unable to take care of the living needs of every old man, thus affecting the satisfaction of the elderly.

The occupation of different old men before entering into the nursing home has a significant difference with the satisfaction. The satisfaction rates of the public institution's retirees and the farmers are relatively high, accounting for $90.9 \%$ and $100.0 \%$ respectively. Through the in-depth interview, we find that causes are not the same. The public institution's retirees do not fear of attacks from behind because they have the living security of the pension and relatively stable source of funding, as well as relatively high cultural quality and strong self-control sentiment ability, thus having a higher life satisfaction; the farmers are experienced much and tough in society with a relatively strong environmental adaptation ability and without too many requirements on the material life, so they are prone to be self-contained and satisfied. Compared with the satisfaction rate of different age groups and the respondents with different degrees of education, there is no significant difference, which is inconsistent with the research result of other domestic scholars ${ }^{[5]}$. The reasons may be about regional differences, sample differences and differences in the nature of institu- 
tions. For the research objects of other domestic scholars, the age group is more concentrated in about 60 to 80 years old. The respondents of this research are mainly the elderly with an age of over 80 . The illiteracy rate among this population is high, and the elderly at this age fail to go to school due to many factors in life. The elderly in that period go through more ups and downs, but they are relatively optimistic, open-minded and have relatively strong environmental adaptation ability, so the research result is different.

\subsubsection{Impact of the service level of nursing home on the satisfaction rate of the elderly}

The service target of the nursing home is to meet multi-level service needs of the elderly, safeguard their legitimate rights and interests, respect their dignity and improve their quality of life. The previous researches of the scholars find that, the living condition, diet level and interpersonal relationship in the nursing home have greater impacts on the satisfaction rate of the elderly ${ }^{[6]}$, which are consistent with the survey results.

Compared with the diet level of different nursing homes, this factor has a greater impact on the satisfaction rate of the elderly. The satisfaction rates of the respondents who feel that the diet tastes "very delicious" and "relatively delicious" are respectively $85.7 \%$ and $93.3 \%$, significantly higher than that of the elderly who feel that the diet tastes "general" and "bed" (P $<0.01)$. In 84 nursing homes surveyed, only the elderly in six institutions have the right to choose diet. Here, the elderly have a card for meal, and report the diet in advance of one week. In this way, the independent choice of the elderly makes them get a lot of satisfaction and meets the needs of the elderly; the diet in the remaining nursing homes remains the same every week. The elderly have no right to choose diet. And some old people even have bad teeth, so they can just keep their body and soul together without concern about the satisfaction rate.

Similarly, the accommodation condition of the nursing home also has a significant impact on the satisfaction of the elderly. The proportion of the elderly who feel that the accommodation condition is "very comfortable" (90.9\%) is higher than that of "general" $(75.1 \%)$ with a significant difference $(\mathrm{P}$ $<0.01$ ); the elderly who feel that the service attitude of the service personnel in the nursing home is intimate and timely account for $86.3 \%$, and the elderly who feel that the service attitude is not intimate and timely account for $50.0 \%$ with a significant difference (P $<0.05$ ). The accommodation condition of the nursing home not only includes indoor environment, but also includes outdoor environment. The interview finds that, the elderly hope that the nursing home can provide a quiet and clean accommodation condition. If there are parks or fitness equipment and other facilities nearby the nursing home, the satisfaction rate of the elderly who live in will be significantly increased.
Furthermore, the indoor environment is directly related to the pension costs paid. In the major nursing homes of Zhengzhou City, the elderly generally live in a double room or a triple room. Only with the bed fee of more than 2,000, the treatment of a single room can be enjoyed. However, most of the elderly choose a multiple bedded room to take care of each other and reduce loneliness. Even if there is friction, the elderly can regulate themselves and live in harmony.

Whether the nursing home can timely treat the disease has a great impact on the satisfaction of the elderly. The satisfaction rate for the nursing home that can timely treat the disease $(97.4 \%)$ is significantly higher than that of the nursing home which fails to timely treat the disease $(40.0 \%)$, with a significant difference $(\mathrm{P}<0.05)$. The survey finds that, when the elderly catch a cold or fever-like illness, most of the nursing homes can provide timely medical services; but when the elderly suffer from large and severe illness, individual nursing homes can timely notify the families of the elderly to avoid delay treatment and also maintain a long-term cooperation with the hospitals so that it is not only convenient for timely treatment of the elderly, but also conducive to good health of the elderly. The interview finds that, the ideal later life of the elderly includes good health, filial children and comfortable life. Thus, the improvement of the medical facilities in the nursing home occupies a pivotal position in the life of the elderly. Meanwhile, more attention shall be given to the subjective health feeling of the elderly to increase awareness of common diseases and diseases suffered by the elderly, so as to help the elderly treat the disease with an objective and mild mentality.

\subsubsection{Impact of the interpersonal relationship on the satisfaction of the elderly}

As a person with dual attributes, the social interaction is an important part of life. The social conditions directly affect people's self-evaluation. And the social support is positively correlated to the mental satisfaction. The survey finds that, the elderly who regularly participate in social activities only account for $7.4 \%$, the elderly who occasionally participate in social activities account for $21.3 \%$, and the elderly who never participate in social activities account for $71.3 \%$. It indicates that the activities of the elderly are relatively simple and few. More than half of the respondents $(71.3 \%)$ do not participate in social activities, which is not very conducive to physical and mental health of the elderly. The in-depth interview finds that, the main social activities of the elderly are seeing a play, play cards, doing setting-up exercise, dancing and so on. Different old people have different awareness of the social activities: $62.0 \%$ of the elderly think that it is meaningful to participate in social activities; $5.6 \%$ think that it is very meaningful to participate in social activities and have their own interests; $12.0 \%$ think that it is meaningless to participate in social activities; 


\section{SHS Web of Conferences}

$21.3 \%$ think it does not matter. However, whether the elderly participate in social activities, whether the volunteers come to visit and the frequency of offspring's care do not have significant differences with the satisfaction, which is different from the research result of the related literature ${ }^{[7]}$. The reasons are as follows: 1) The respondents are mostly over 70 years old $(88.0 \%)$ with a high age and without excessive requirements on the volunteers and social activities, and their vitality, enthusiasm for life, energy and maintaining a good interpersonal relationship are worse than that of the elderly with a low age. 2) $62.0 \%$ of respondents feel that it is meaningful to participate in activities, indicating that most of the elderly still have the will to participate in activities. However, in the survey, the elderly who can partly take care of themselves account for $25.9 \%$, so the proportion of the elderly who never participate in activities is very high (46.3\%); moreover, the nursing homes take into account the safety of the elderly, so they will also reduce the frequency of social activities. The elderly with a high degree of education who can quickly adjust their emotions in a noisy or silent environment often have their own interests and hobbies in life, such as reading, writing biography, playing chess and listening to music, so as to adapt as quickly as possible. Therefore, they can entertain themselves even with a low frequency of child visit, few social activities and rare visit by the volunteers, so it does not have a significant impact on the satisfaction. 3) Child care is the consolation for the soul of the elderly. However, in the survey, the elderly keep an indifferent attitude towards child visit. It is unable to realize high expectations for the children. Some old people come to the nursing home because they have a bad relation with family members. Even if the children come to visit, the satisfaction will also not increase. Most of the elderly do not want to increase the burden on their children and think that their work is busy, so the elderly do not have too many requirements, and their children can arrange visit according to spare time.

Of course, the in-depth interview also finds that, the elderly still hope that the volunteers can regularly visit, accompany with them to chat, walk and organize performances. The respondents who hope the volunteers can regularly visit account for $70.4 \%$. It indicates that the inner world of the elderly is lonely; they are required to be concerned by all aspects of society.

\section{SUGGESTIONS ON FURTHER ENHANCEMENT AND IMPROVEMENT OF SERVICE QUALITY IN NURSING HOMES}

The research shows that, most of the elderly in the nursing homes are quite satisfied with the current life and keep an optimistic attitude towards life, but a considerable number of respondents have no choice but to be content with the status quo. Under the back- ground of weakening support of family and accelerated aging population, the institutional care has become an inevitable trend. According to the research result, how to make the nursing homes win the hearts of all shall be improved from the following aspects:

First, specialize, standardize and improve the service quality of nursing homes. The nursing homes continuously improve and meet the needs of different levels of the elderly through specialized and standardized social pension service system, so the elderly can step on a low-cost pension road integrated with public welfare and marketization. On the diet, the types of meals shall be rich as much as possible on the basis of ensuring food safety and hygiene, and the meals shall be suitable for the taste of the elderly, soft and easy to digest so that the elderly have the right to independently choose and improve their self-acceptance. On the accommodation, it shall meet the needs of the elderly and let the elderly with the same disease live together, so as to reduce quarrels due to misunderstanding of the pain; fully consider the economic affordability of the elderly and increase the quantity of single rooms and reduce the cost of single rooms as much as possible; arrange the elderly who are inconvenient to act in the lower floor, so as to facilitate the elderly to do outdoor activities.

Second, seek for governmental and social assistance in funds and policies actively. The survey finds that the nursing staff generally has a poor remuneration, low social status and large labor intensity, so some youths feel it's not promising to be a nursing staff in long term, and they are unwilling to engage in this industry which hence lacks manpower. Many nursing homes have problems in recruiting the nursing staff. Nowadays, most of the nursing staffs that come from the countryside are at the age of 50 to 60 without related experience just provide nanny-typed care, so there is a weird phenomenon: "the elderly care for the elderly". Therefore, the nursing homes should actively raise funds, and the relevant functional departments should introduce related policies to jointly safeguard the legitimate rights and interests of the nursing staff and increase their wages, welfare guarantee, and provide professional skills training, and constantly improve the quality of the nursing staff, so the nursing homes can attract and retain people; in addition, the nursing homes should increase social propaganda and acceptance of the society and the masses so that more people understand the importance and the difficulties of the pension undertakings and offer supports from different aspects.

Third, build a family-typed nursing home which supports the elderly and warm their hearts with family ties, and realize "the homely nursing home". However, the requirement toward the family affection of the nursing home is much higher than that of the humanization. It is difficult to achieve for each nursing home, which is a very high standard to endow the function of the "family support" in the nursing home. The elderly 
also have human dignity and need to be respected and beloved. The family affection of the nursing home is to let the elderly feel the warmth of home, and ensure human dignity and quality of life of the elderly.

\section{ACKNOWLEDGEMENT}

This paper is financially supported by Key Project of the Higher Educations and Institutions of Henan Province, China (GN: 15B180002) and Fundamental and Frontier Science Technology Research of Henan Province, China (GN: 152300410092).

\section{REFERENCES}

[1] Yan Bingqiu \& Gao Xiaolu. 2013. Home care satisfaction analysis of aging urban Chinese populations: potential factors and community difference. Geographical Research, 32(7): 1269-1279.
[2] Li Juan, Yang Ruizhen \& Yang Xiao, et al. 2015. Analysis of the elderly satisfaction on nursing service in different types of nursing homes. Chinese Health Industry, 16: 75-77.

[3] Qiu Di. 2015. Research report of the elderly satisfaction on nursing service in urban community. Value Engineering, 12: 257-259.

[4] Tang Qiqun. 2014. Analysis of pension mode and satisfaction influencing factor of the elderly in Hebei Province. Chinese Journal of Coal Industry, 17(12): 2091-2094.

[5] Zhang Huijun, Huang Fei \& Yin Jiao. 2011. Nursing service satisfaction and needs of the elderly in nursing home in Liaoning Province. Chinese General Practice, 22: $98-105$.

[6] Li Dan, Xu Xin \& Guo Zhenyou. 2013. Study on happiness among the elderly in apartment for the aged in Tangshan. Modern Preventive Medicine, 40(19): 3633-3635.

[7] Huang Fei, Zhang Huijun \& Yin Jiao. 2012. Current status and demand of nursing staff training in the nursing home in Liaoning Province. Chinese Journal of the Elderly, 2 (32): 571-573. 